\title{
LE CHANGEMENT DE RYTHME DE LA GLOBALISATION PEUT-IL ETRE UNE CHANCE POUR LES ÉMERGENTS LATINO-AMÉRICAINS?
}

\section{Pierre Salama ${ }^{\prime}$}

\begin{abstract}
Résumé
L'article analyse le récente croissance du commerce international des biens et services et la mise plus d'ouverture des pays au commerce international et le fin de la globalisation à partir de 2008, avec la reprise des politiques protectionnistes dans de nombreux pays, en particulier aux États-Unis. Dans cette conjoncture préconise que le ralentissement du commerce international et la montée du protectionnisme peut être une opportunité pour les pays émergents d'Amérique Latine à prendre un autre modèle de développement et une croissance plus sustenable, qui respecte les citoyens et l'environnement. Il dit que, dans ce contexte, de nouvelles alliances de classe peuvent se produire, comme dans les années trente, et permettre la reprise économique dans ces pays, dans le reflux de la globalisation.
\end{abstract}

Mots-clés: Reflux de la globalisation ; Politiques protectionnistes ; Autre modèle de développement sustenable; Nouvelles alliances de classe ; Reprise économique.

Le commerce international des biens et des services s'est accru deux à trois fois plus rapidement que le PIB mondial des années 1990 à la crise de 2008. En moyenne, tous les pays sont donc davantage ouverts qu'ils l'étaient par le passé. Cependant, certains pays se sont davantage ouverts. Lorsqu'on compare, par exemple, le poids des exportations du Brésil et de la Chine dans les exportations mondiales au début des années 1980, on se observe qu'il est à peu près équivalent dans les deux pays, soit 1\%. Une trentaine d'année plus tard, les exportations brésiliennes s'élèvent à 1,1\% des exportations mondiales, alors qu’en Chine elles atteignent $12 \%$. Les deux pays se sont donc ouverts, mais à des rythmes très différents.

Depuis 2008, malgré une très légère reprise économique dans les pays avancés, la globalisation semble s'essouffler. Les exportations mondiales croissent désormais à un rythme proche de celui du PIB mondial. Des mesures protectionnistes se multiplient depuis 2012. Avec, l'accession de Trump à la présidence des Etats-Unis (2017), elles pourraient à la fois être plus importantes et se généraliser.

Dans ces conditions, quelles sont les possibilités pour les pays latino-américains de faire face à ces

\footnotetext{
${ }^{1}$ Professeur émérite des universités Centre d'Économie de Paris-Nord CEPN - CNR. Directeur scientifique de la Revue Tiers Monde et du Groupe de Recherche sur l'État, l'Internationalisation des Techniques et le Développement (GREITD). E-mail: psalama@wanadoo.fr
} 
menaces ? Le ralentissement de la croissance des pays latino-américains depuis 2012, voire la crise profonde traversée par le Brésil et dans une moindre mesure l'Argentine, peut-il être une opportunité de s'insérer «positivement» dans la division internationale du travail en suscitant des exportations autres que les matières premières? A vrai dire cette possibilité parait faible, mais elle existe.

\section{UN BASCULEMENT DE LA DIVISION INTERNATIONALE DU TRAVAIL}

\section{Des économies relativement fermées et des modes d'industrialisation originaux}

\section{- Une croissance tirée par le marché intérieur en voie de formation en Amérique latine}

Suite à la grande crise de 1929, entre 1935 et 1980, quelques pays d'Amérique latine ont connu une industrialisation conséquente connue sous le nom de substitution aux importations, ou encore de croissance tirée par un marché intérieur en voie de formation. Celle-ci se caractérisait par la capacité à intégrer le plus possible les activités en amont et en aval.

En Amérique latine, dans les grands pays, L’industrialisation a été relativement incomplète, voire «tronquée » selon l'expression utilisée par Fajnzylber F. ${ }^{2}$ : malgré une intervention massive de l'Etat dans les secteurs lourd, les chaines productives n'ont pu être entièrement intégrées faute de capacités financières, humaines, techniques suffisantes. C'est pourquoi on les a qualifiées à l'époque d'économies semi-industrialisées. Dès la fin des années 1970, les difficultés s’accumulent : l'industrialisation est moins dynamique, la croissance du PIB est très volatile, l'hyperinflation (ou une inflation très élevée) s'enracine, la pauvreté augmente de nouveau et les inégalités dans la distribution des revenus tendent à s'élever alors même qu'elles se situent déjà à un niveau très élevé. Dans les années 1990, la hausse des prix est maitrisée mais la croissance retrouvée demeure à un niveau modeste surtout si on la compare à celle que connaissent plusieurs pays asiatiques.

\section{$\underline{\text { Une croissance tirée par les exportations en Asie sous certaines conditions }}$}

Dans les années 1960 à 1980, quelques pays asiatiques ${ }^{3}$ vont connaitre une industrialisation rapide grâce à la fois à des délocalisations et une intervention de l'Etat importante. Les délocalisations concernaient certains segments de la ligne de production d'un produit pour lesquels le coût unitaire du travail (combinaison du taux de salaire, de la productivité et du taux de change) était largement inférieur à celui des pays avancés. Plus précisément, à l'origine, ces délocalisations concernaient peu de produits pour lesquels il était possible d'utiliser des

\footnotetext{
${ }^{2}$ Fajnzylber F. (1980), Industrializacion e internationalizacion de la America Latina, Fundo de Cultura economica, Mexico

${ }^{3}$ (les dragons : Corée du Sud, Taïwan, Singapour et Hong-Kong, suivis par les tigres : Malaisie, Thailande, Indonésie, etc.,)
} 
technologies alternatives, dites labour using, utilisant peu de capital et une main d'œuvre abondante et peu rémunérée. L'écart de salaires entre ces pays du Sud et ceux du Nord, la possibilité d'imposer des conditions de travail inenvisageables dans les pays avancés compte-tenu de leurs législations, surcompensait l'écart de productivité de telle sorte que la délocalisation au Sud de certains segments de production devenait plus rentable que leur production au Nord, malgré les coûts induits par des transports trancontinentaux. Les salaires augmentant par la suite dans ces pays, l'intensité capitalistique a suivi et le nombre de produits concernés par les délocalisation s'est élargie, et ce d'autant plus rapidement que les gouvernements des pays asiatiques, à l'exception notable de Hong-Kong, favorisaient à la fois une intégration nationale par l'amont, mettait à la disposition des entreprises une main d'œuvre de plus en plus qualifiée, adoptaient une politique industrielle conséquente visant à s'insérer de manière positive dans la division internationale du travail, cherchant à produire des biens plus sophistiqués davantage demandés sur les marchés internationaux. L’essor de cette segmentation-délocalisation, parfois désignée comme sous-traitance internationale, concernait deux a trois acteurs: une entreprise des pays avancés donneuse d'ordre, une à deux entreprises à la périphérie, celles-ci pouvant être une filiale de l'entreprise des pays avancés, mais pas nécessairement comme cela a été le cas surtout en Corée du Sud.

Dans les années 90 et surtout 2000, on a assisté à un bouleversement de la division internationale du travail avec le développement de l'internet, la baisse du coût des transports, la possibilité que certains pays puissent adapter leur offre très rapidement aux brusques changements de la demande mondiale. On est passé d'une relation entre deux à trois acteurs à une relation entre un acteur, le donneur d'ordre et $\ll \mathrm{n} »$ acteurs situées dans différents pays, surtout au Sud mais également au Nord. La chaine internationale de valeur éclate alors, la ligne de production se situant dans « $\mathrm{n} \gg$ pays de la conception à la distribution en passant par l'ensemble des segments. Les techniques de production changent, y compris dans l'assemblage. En effet, l'avantage du faible coût du salaire des travailleurs peu qualifiés devient insuffisant par rapport aux gains procurés par l'utilisation de techniques de production plus sophistiqués, capital intensives, utilisant une main d'œuvre plus qualifiée et plus coûteuse mais relativement moins chère que dans le Nord. C'est de cette manière, qu'au-delà de la relation Nord-Sud portant sur des biens manufacturés, se sont tissées des relations Sud-Sud de plus en plus denses qu’on a parfois qualifiées de révolution silencieuse.

\section{Les pays latino-américains peu investis dans la chaine internationale de valeur}

Comme le remarquent Hiratuka et Sarti ${ }^{4}$ à la suite de Baldwin ${ }^{5}$, avec l'éclatement international de la chaine de valeur dans les années 2000, la poursuite de l'industrialisation aurait pu être impulsée en Amérique

\footnotetext{
${ }^{4}$ Hiratuka et Sarti (2016), «Relações econômicas entre Brasil e China: análise dos fluxos de comércio e investimento direto estrangeiro », Revista Tempo du Mundo, Brasil, IPEA, vol 2 n 1, 2016, pp.83-98
} 
latine à deux conditions: 1/ rendre attrayante la possibilité de produire des segments de production; 2/ entreprendre comme ce fut fait en Asie une politique industrielle qui permette d'intégrer pour partie en amont ces segments délocalisés et remplacer des inputs importés par des segments produits localement répondant aux exigences internationales de haute qualité. Ce fut peu le cas en Amérique latine, si bien que les grandes économies latino-américaines ont relativement peu participé au processus d'éclatement international de valeur que ce soit en amont ou en aval ${ }^{6}$, y compris au Mexique. Le Mexique en effet, à la différence de nombre de pays asiatiques, s'est cantonné pour l'essentiel à des activités d'assemblage à l'exception en partie de certains secteurs comme l'industrie automobile où le nombre d'équipementier a augmenté. L'ouverture croissante n’a pas eu d'effets positifs sur la croissance, les effets multiplicateurs sur le PIB étant de ce fait faibles ce qui explique que parmi les grands pays latino-américains, il ait été celui dont la croissance à été la plus faible ces vingt cinq dernières années? ${ }^{7}$ La complexification de son tissu industriel est également faible et ou/bien apparente et trompeuse ${ }^{8}$.

\section{La révolution numérique, la baisse du coût des transports, la flexibilité des emplois ont stimulé ce basculement}

\section{Une révolution.}

Hier, les pays dits de la périphérie étaient spécialisés dans la production de matières premières. Aujourd'hui, certains d'entre eux, en Asie et au Mexique, sont devenus des « ateliers du monde », d'autres, après s'être industrialisés, principalement en Amérique latine sont revenus vers une spécialisation portant sur la production de produits de rente et se sont de ce fait reprimarisés, d’autres enfin, sont restés spécialisés dans la production de produits primaires.

Les bouleversements observés concernent également les pays avancés. Leurs lignes de production se

\footnotetext{
${ }^{5}$ Baldwin R (2016), The Great convergence, Information, Technology end the New globalization, Harvard University press.

${ }^{6}$ La plupart des pays latino-américains sont peu intégrés dans les chaînes internationales de valeurs. La CEPAL (Commission économique pour l'Amérique latine) distingue deux types d'intégration: par l'amont (backward), qui mesure pour un pays donné la part des biens intermédiaires importés, incorporés dans ses exportations, et par l'aval, qui mesure la part des biens intermédiaires exportés par un pays qui sont incorporés dans les exportations d'autres pays. La participation par l'amont est de 11,4\% en 2000 et 10,7 \% en 2011 au Brésil au lieu de respectivement 37,2 \% et de 32,1\% pour la Chine. La baisse du ratio chinois est révélatrice de l'effort de la Chine pour intégrer ses lignes de production. La participation par l'aval est plus importante pour le Brésil (17,1 \% en 2000 et 24,5\% en 2011) que pour la Chine (10,8\%et 15,6\%) car le Brésil exporte plus des matières premières vers la Chine qui les incorporent dans ses exportations, voir: OCDE (Organisation de coopération et de développement économiques), CAF (Corporacion Andinade Fomento) et CEPAL (2016), Latin American Economic Outlook (2016), Towards a New Partnership with China.

${ }^{7}$ Voir : Romero Tellaeche J. A. (2014), Los limites al crescimiento economico de Mexico, El Colegio de Mexico, Universidad national autonoma de Mexico.

${ }^{8}$ Sur les soixante dernières années, voir Salama P.(2012), Les économies émergentes latino-américaines, entre cigales et fourmis, édition Armand Colin, et Salama P (2016), Amérique Latine, des années 1950 à aujourd'hui, mutations, essor et nouvelles dépendances, revue d'économie financière, $n^{\circ} 124,23-44$
} 
sont davantage internationalisées, se concentrant souvent sur les segments en amont, ceux où le coefficient recherche -développement et l'intensité capitalistique sont les plus élevés, et en aval. Les autres segments étant en partie délocalisés vers d'autres pays. C’est ce mouvement que l'OCDE caractérise comme un «smile», un sourire.

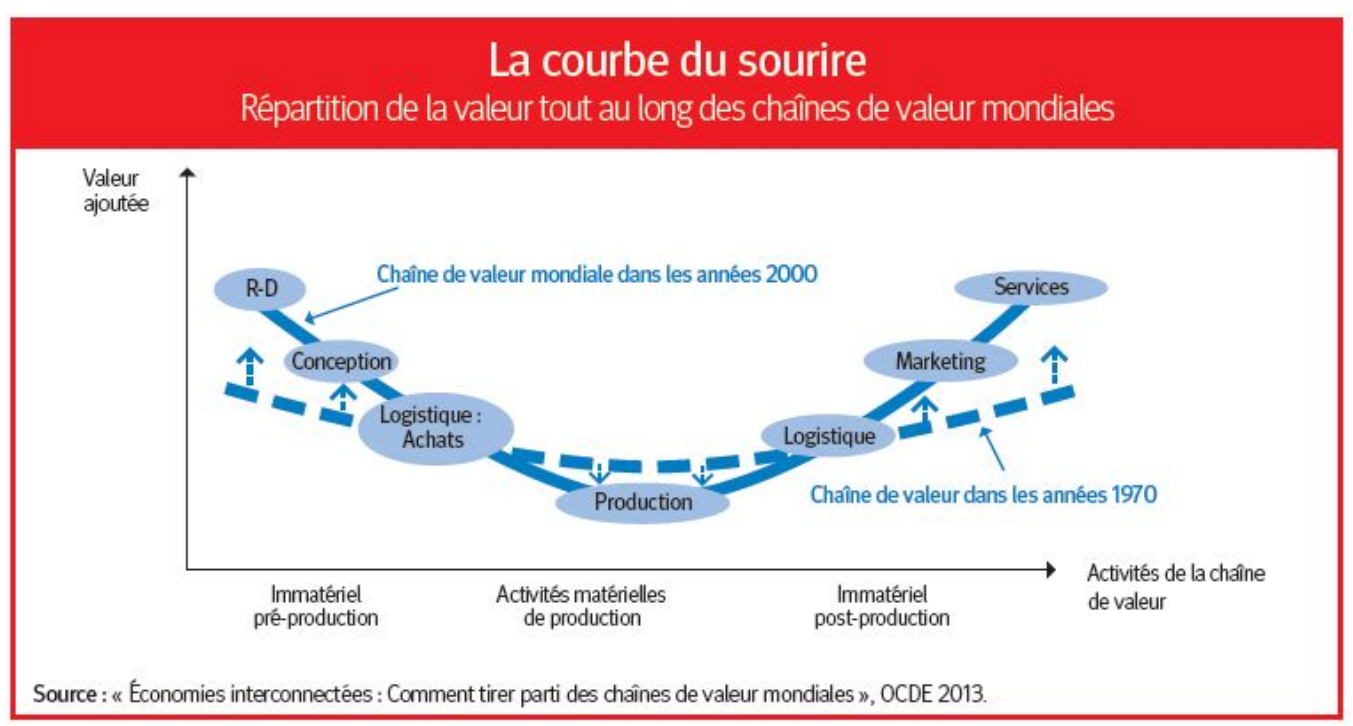

Avec la révolution internet et la numérisation, les frontières entre l'industrie et certains services sont devenues poreuses. D’abord parce que toute une série d'activités qui hier faisaient partie de l'industrie ont été externalisées et sont aujourd'hui désignées comme services, ce qui rend parfois les discussions sur la désindustrialisation peu pertinentes lorsque les comparaisons ne sont pas faites a périmètre équivalent. Ensuite parce que pour les services dits dynamiques, les plus performants, ceux dont la productivité est non seulement élevée mais croit rapidement, les méthodes qui y sont appliquées sont celles de l'industrie, celles-ci se diffusant dans ceux là.

La baisse du coût des transports, la capacité de monter en échelle très rapidement, c'est-à-dire la capacité de s’adresser à des marchés immédiatement mondiaux et de mobiliser des ressources financières et humaines très importantes pour le faire (scalabilité), enfin la flexibilisation de la force de travail, permettent une adaptabilité rapide le d'offre aux mouvements de la demande mondiale. Cet éclatement de la chaine internationale de valeur concerne surtout des pays asiatiques. Les relations commerciales entre eux deviennent encore plus denses qu'elles l'étaient déjà, avec pour destin final les marchés des pays avancés.

La transnationalisation de la production des pays avancés ne se limite pas aux marché extérieurs à la Périphérie, mais aussi à leurs marchés internes, avec en Chine principalement des règles et des modalités différentes de celles en vigueur pour la production destinée à l'exportation. La transnationalisation d'entreprises de quelques pays de la périphérie, principalement la Chine, obéit à d'autres logiques : les investissements massifs de la 
Chine à l'étranger ont pour objet principalement de s'assurer l'accès aux matières premières tant pour la production que pour leur transport, de s'accaparer des dernières technologies produites des entreprises des pays avancés grâce à leur achat, secondairement de produire pour les marchés intérieurs tant des pays semiindustrialisés que des pays avancés.

Pour toute une série de produits, les formes de la concurrence reposent de plus en plus sur la maitrise de l'amortissement des coûts fixes, en particulier des coûts de conception et d'investissement, comme le souligne Pierre Veltz (p.67) ${ }^{9}$. L'industrie du logiciel en est un exemple presque parfait puisque les coûts de reproduction sont voisins de zéro (le coût marginal nul pour reprendre l'expression de Jeremy Rifkin ${ }^{10}$ ), par contre les coûts en amont sont extrêmement élevés (les infrastructures d’internet comme le cloud) et rendent très difficiles pour des pays émergents de s'imposer sur ces produits très dynamiques. L'accès à ces secteurs exige de très gros efforts en recherche et la capacité d’atteindre une échelle de production considérable, ce qui constitue une «barrière à l'entrée » très forte. Ce n'est qu'une fois franchie celle-ci que les profits arrivent.

Avec la révolution internet, les économies d’agglomération ont acquis davantage de forces que par le passé. La numérisation éloigne et rapproche, ce qui à première vue semble être un paradoxe. Eloigne, parce qu’elle permet un éclatement international de la chaine de valeur, rapproche, parce que les grandes villes acquièrent une puissance économique considérable au point qu’elles paraissent être les «gagnants» de la mondialisation, au détriment des moyennes et petites villes, devenues les «perdants » de celle-ci. S. Sassen ${ }^{11}$ avait déjà analysé la montée en puissance des grandes métropoles au début des années 1990. Les économies d’agglomération ont pris une ampleur considérable parce que les grandes villes sont à la fois des bassins de demande, de fournisseurs, de main d'œuvre formées et qualifiés considérables. Autour des grandes villes se constituent des puissances économiques qui, établissant souvent des réseaux entre-elles au-delà des frontières nationales, s'opposent parfois aux intérêts définis par les gouvernements nationaux et. Les grandes villes, à certaines conditions, sont donc le lieu où se développe la complexité, source d’insertion positive dans la division internationale du travail.

\footnotetext{
${ }^{9}$ Veltz P. (2017), La société hyper-industrielle, le nouveau capitalisme productif, édition du Seuil

${ }^{10}$ Rifkin J (2016), La nouvelle société du coût marginal zéro, Línternet des objets, l'émergence des communaux collaboratifs et l'éclipse du capitalisme, édition Babel

${ }^{11}$ Sassen S. (1991), The Global City: New York, London, Tokyo Priceton University Press
} 


\section{Mesure de la complexité économique}

La complexité d'une économie dépend de l'effort effectué en recherche développement, plus celui-ci est élevé, plus la probabilité que l'économie puisse produire des produits complexes est élevée. Les exportations ont deux caractéristiques: leur ubiquité et leur diversification. L’ubiquité dépend de la rareté, laquelle dépend soit des ressources naturelles que le pays a ou n'a pas, soit de la capacité à produire des biens sophistiqués que seuls quelques pays peuvent faire. Afin d'isoler cette dernière et construire un indicateur de complexité, les auteurs, cités ci-dessous, cherchent à utiliser la diversité des exportations pour mesurer le degré d'ubiquité et donc de complexité. Donnons un exemple: le Pakistan et Singapour ont un PIB semblable et exportent chacun 133 grands produits, la diversité de leurs exportations est donc semblable, mais les produits exportés par le Pakistan le sont également par 28 autres pays, dont les exportations sont par ailleurs peu diversifiées. Tel n'est pas le cas de Singapour: seuls 17 autres pays exportent des produits semblables aux siens et leurs exportations sont très diversifiées. Le degré de complexité de l'économie de Singapour est donc plus élevé que celui du Pakistan, dont le revenu par tête est largement inférieur. A partir de ces deux variables: diversité et ubiquité, on peut construire un indicateur.

Pour l'analyse de la complexité voir les travaux de Hausmann et alii (2004) : «Growth Accelerations », mimeo Harvard, Hausmann, Hidalgo et allii (2014), The Atlas of Economic Complexity, Mapping Paths to Perspectives, Center for International Development, Harvard University, Harvard Kennedy School, Macro Connections MIT Media Lab, et plus récemment, reprenant la méthodologie de Hausman et de Hidalgo, voir Paulo Gala (2017), Complexidade economica, uma nova perspectiva para entender a antigua questao da riqueza das nacoes, editions: Centro International Celso Furtado et Contraponto, d'où nous avons pris l'exemple du Pakistan et de Singapour ( p.21-26).

Plus une économie est complexe, plus elle produit des biens sophistiqués nécessitant un fort coefficient de recherche et voce et versa. C'est une tautologie. Ce sont ces produits qui ont une élasticité de la demande par rapport au revenu élevée et par rapport aux prix, faible. Ce sont des produits qui permettent une insertion positive dans la division internationale du travail parce qu'ils constituent un pari sur l'avenir. Peu de pays du Sud parviennent à favoriser une complexité croissante de leur tissu industriel. Ceux qui y parviennent (les dragons comme la Corée du Sud, Taïwan ... la Chine depuis quelques années) délocalisent en partie leur production de biens labour using utilisant des techniques de production peu sophistiquées dans des pays encore moins avancés qu'eux (Bengladesh, Vietnam, etc.) caractérisés par de salaires encore plus faibles et des conditions de travail dignes de la naissance du capitalisme dans les pays avancés.

Conséquences à la fois de la numérisation, de la baisse des coûts de transport, de la flexibilité et de la scalabilité possibles, les technologies de pointe sont immédiatement disponibles. Dit autrement, est révolue le temps (les années 60 à 90) où on pouvait exporter du capital productif dévalorisé dans les pays avancés, valorisables à la périphérie comme ce fut le cas dans l'industrie automobile où Ford, Fiat, Renault, Peugeot, Volkswagen ,etc.. continuaient à produire des vielles voitures « neuves $\gg($ coccinelle, falcon, etc..). Aujourd'hui le niveau de productivité atteint par les firmes multinationales dans les pays émergents est proche de celui des pays avancés. Compte-tenu des salaires plus faibles et parfois malgré une tendance à l'appréciation des monnaies nationales face que dollar, elles sont souvent plus compétitives que les entreprises restées dans les pays avancés. Le cas de l'industrie automobile au Mexique le montre nettement. Le monde n'est plus constitué de strates à la Vernon (les cycles de produit), mais de réseaux, il est devenu un archipel comme le souligne P.Veltz. 
$\underline{\text { Des conséquences importantes sur l'emploi et ses formes, sur la représentation politique }}$

On ne saurait omettre les conséquences sur le travail, l'emploi, la représentation politique au prétexte que celles-ci feraient appel à d'autres disciplines que l'économie. La globalisation est à la fois un processus d'unification et de fragmentation dans les pays avancés. Unification parce qu'avec l'ouverture croissante l'emploi et le travail sont soumis à des contraintes externes de plus en plus élevées. L’emploi tend à devenir plus précaire et avec l'essor d'internet, l' «ubérisation » des activités devient de plus en plus importantes. Elle se manifeste par un essor de la tertiarisation. Le travailleur, devenant son propre employeur, est inséré dans des contraintes qui s'imposent à lui en amont grâce au cloud et au big data que maitrisent seules des grandes sociétés. Fragmentation parce que toutes les activités ne sont certes pas sujettes à une tertiarisation. Ce mouvement récent fragmente encore plus l'ensemble des travailleurs que par le passé ou s'opposaient les insiders et les outsiders, avec le déclassement et la surtout la désaffiliation qui vont avec. Le travail tend à devenir de plus en plus flexible tant au niveau des rémunérations (concurrence des bas salaires asiatiques et effets de la précarité sur les salaires) que de la polyvalence des tâches sans que les possibilités de mobilité sociale soient suffisamment satisfaites en cas de licenciements. A cela s'ajoute un écart croissant dans les rémunérations du travail entre les tâches d'exécution et celles de direction, auquel s'ajoutent les effets inégalitaires produits par la libéralisation financière. La représentation politique est fortement remise en question par ce mouvement au niveau de l'emploi - tant au niveau quantitatif que qualitatif - et des salaires. Responsables de la globalisation qu'elles ont favorisée, elles en paient le prix aujourd'hui en raison de leur incapacité avérée de la maitriser et laissent la place à de nouvelles configurations politiques dont les contours sont difficiles à prévoir.

\section{DES ÉCONOMIES LATINO-AMÉRICAINES AFFAIBLIES MAIS QUI CONSERVENT DES POSSIBILITÉS DE « REBONDIR »}

\section{Un tissu industriel fragilisé}

Les grands pays latino-américains plus protectionnistes que les pays asiatiques par les droits de douane mais moins par les taux de change.

Nous avons vu que les principaux pays latino-américains se sont ouverts au commerce mondial de marchandises à un rythme plus modéré que les pays asiatiques (à l'exception notable de l'Inde jusque récemment). Le protectionnisme, en baisse, reste relativement élevé au Brésil et en Argentine surtout si on le compare à la plupart des pays asiatiques y compris sur les biens d'équipement et sur les produits intermédiaires. C'est un paradoxe, car l'idéologie dominante en matière de commerce extérieure est plutôt celle du libre-échange. 
Tableau $n^{\circ} 2$ : Tarifs moyens d'importations sur les vingt dernières années

\begin{tabular}{|c|c|c|c|c|c|c|c|c|c|}
\hline & Mexique & Argentine & Brésil & Pérou & Chili & Colombie & $\begin{array}{c}\text { Corée du } \\
\text { Sud }\end{array}$ & Chine & $\begin{array}{c}\text { Etats- } \\
\text { Unis }\end{array}$ \\
\hline $\begin{array}{c}1996 \text { ou } \\
1997\end{array}$ & 14.8 & 14.5 & 14.4 & 13.2 & 11 & 12.2 & 9.8 & 22 & 4.1 \\
\hline $2015^{*}$ & 3 & 12.5 & 13.7 & 2.8 & 1.2 & 5.2 & 5.2 & 7.6 & 2.8 \\
\hline \multicolumn{3}{|c|}{ Source : Banque mondiale, annexe statistique et Bradesco, ${ }^{*}$ ou donnée la plus recente } \\
\hline
\end{tabular}

Tableau 3: Tarifs moyens sur les biens d'équipement et les produits intermédiaires

\begin{tabular}{|c|c|c|c|c|c|c|}
\hline & \multicolumn{3}{|c|}{ Biens de capital } & \multicolumn{3}{c|}{ Biens intermédiaires } \\
\hline PAYS & 2000 & 2006 & 2010 & 2000 & 2006 & 2010 \\
\hline Brésil & 16.90 & 13.20 & 13.00 & 13.90 & 10.70 & 11.70 \\
\hline Chine & 14.40 & 8.10 & 7.70 & 14.40 & 7.90 & 7.40 \\
\hline Corée & 7.20 & 5.90 & 6.00 & 8.00 & 11.20 & 11.10 \\
\hline Philippines & 4.20 & 2.80 & 2.90 & 5.90 & 5.00 & 4.90 \\
\hline Inde & 26.70 & 14.10 & 8.40 & 32.70 & 17.40 & 10.00 \\
\hline Indonésie & 4.40 & 3.80 & 5.50 & 7.30 & 6.10 & 6.00 \\
\hline Malaisie & 5.10 & 4.20 & 3.60 & 7.10 & 6.80 & 6.60 \\
\hline Mexique & 13.10 & 9.50 & 3.20 & 14.80 & 11.80 & 6.10 \\
\hline Thailande & 10.50 & 6.40 & 5.50 & 14 & 6.00 & 4.40 \\
\hline
\end{tabular}

Le protectionnisme est plus important en Amérique latine que dans les pays asiatiques, à l'exception de quelques pays comme le Pérou, le Chili ou encore la Colombie et bien sûr le Mexique. Cependant, l'appréciation des monnaies latino-américaines sur longue durée atténue fortement, voire annihile, les effets protecteurs des droits de douane.

\section{Taux de change réel effectif bilatéral du Brésil de mars 1994 à mars 2016 \\ (base 100 en juin 1994)}

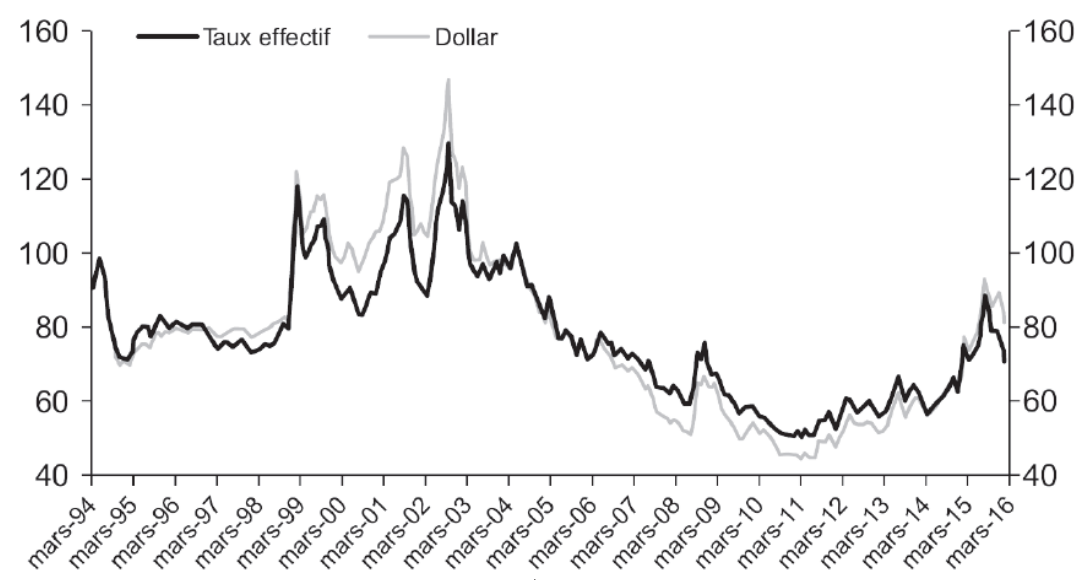

Note : rappelons qu'une courbe décroissante signifie une appréciation de la monnaie nationale par rapport au dollar américain. Les taux de change sont exprimés en termes réels afin de tenir compte des différentiels d'inflation avec les États-Unis.

Source : Banque centrale du Brésil. 
Cette appréciation réduit le prix des importations en monnaie locale, celles-ci rendues moins chères se substituent pour partie à la production locale pour trois raisons : le niveau atteint par la productivité du travail est relativement plus faible ${ }^{12}$ que celui des entreprises exportant les mêmes produits, la productivité du travail a augmenté faiblement (voir tableau ci-dessous), bien plus faiblement que dans les pays asiatiques, et, enfin, dans les pays ayant eu des gouvernements progressistes, la croissance des salaires réels a cru plus rapidement que celle de la productivité du travail. Ces trois facteurs, auxquels il convient d'ajouter les fortes appréciations des monnaies locales face au dollar, linsuffisance des infrastructures et souvent des institutions peu « transparentes », minent la compétitivité des entreprises, réduit à terme la rentabilité des capitaux engagés, en moyenne, dans le secteur industriel.

\section{Tableau $n^{\circ}:$ Taux de croissance annuel de la productivité du travail dans l'industrie, plusieurs pays}

\begin{tabular}{|c|c|c|c|c|c|}
\hline & $1970-1979$ & $1980-1989$ & $1990-1999$ & $2000-2007$ & $2010-2014$ \\
\hline Argentine & 1.7 & -1.4 & 6.9 & -0.7 & -2.5 \\
\hline Brésil & 3.4 & -2.8 & 2.9 & 0.0 & -2.5 \\
\hline Chili & -0.3 & -0.5 & 6.2 & 0.1 & -1.8 \\
\hline Mexique & 0.6 & -1.7 & 0.4 & 0.6 & 3.7 \\
\hline Chine & -1.6 & 4.8 & 10.4 & 7.1 & 6.9 \\
\hline Corée du Sud & 3.2 & 5.0 & 7.7 & 5.6 & 4.3 \\
\hline
\end{tabular}

Source : UNCTAD (2016), Trade and Development Report, p. 69

\section{$\underline{\text { Une désindustrialisation précoce }} \underline{\underline{13}}$}

L’appréciation de la monnaie nationale, le faible niveau de la productivité et sa progression léthargique, la hausse des salaires réels dans quelques pays, et de manière indirecte, l'insuffisance des infrastructures, expliquent en grande partie la faiblesse de l'investissement dans l'industrie de transformation. Celle-ci précipite la désindustrialisation de ces pays au profit d'activités rentières beaucoup plus lucratives (matières premières,

\footnotetext{
${ }^{12}$ Il faut se défier des moyennes, la dispersion autour de la moyenne est beaucoup plus élevée dans les pays émergents latinoaméricains que dans les pays avancés. Des grandes entreprises, notamment les filiales des transnationales, ont des niveaux de productivité proches de ceux qu'elles ont dans les pays avancés alors même que la productivité moyenne et sa progression peuvent être faibles. Lorsqu'on décompose les entreprises selon leur taille en quatre groupes (grandes, moyennes, petites et très petites) et qu'on compare leur productivité moyenne aux groupes correspondants aux Etats-Unis, indicés 100 pour chacun d'entre eux, on constate que la brèche de productivité n'est pas très importante dans le groupe des grandes (on pourrait ajouter que la productivité des transnationales est plus élevée en général que celle des entreprises nationales, sauf exception), mais qu’elle croit de plus en plus fortement à mesure que l'on passe du groupe de moyennes, à celui des petites et enfin des très petites (où est concentré l'emploi informel). On obtient des résultats comparables pour ce qui concerne les revenus du travail, la dispersion y est très importante. Ocde-Cepal (2012), Perspectives économiques de l'Amérique latine, transformation de l'Etat et développement

${ }^{13}$ On dit que la désindustrialisation d'un pays émergent est précoce par rapport à la désindustrialisation affectant certains pays avancés lorsque le revenu par tête au début de ce processus correspond à la moitié de celui des pays avancés au moment où débute leur désindustrialisation. La désindustrialisation concerne non seulement la baisse relative et absolue des emplois industriels, et la destruction d'une partie du tissu industriel, celle produisant notamment les produits de bas de gamme.
} 
finance).

Au final, les taux de change apprécié, la faiblesse de la productivité du travail et de son évolution, des salaires réels qui, bien que faibles, croissant parfois plus vite que la productivité, ouvrent les pays d'Amérique latine à la concurrence étrangère davantage que le protectionnisme relativement plus élevé l'empêche ${ }^{14}$.

Il existe une relation de causalité entre l'appréciation de la monnaie nationale et la faible progression de la productivité en moyenne. L'appréciation rend plus coûteux le travail exprimé en dollar quand bien même les salaires resteraient stables en monnaie nationale. De ce fait, la rentabilité est affectée et la tendance à s'orienter vers des activités soit de rente, soit spéculatives (immobilières), soit financières, ces dernières pouvant être de caractère spéculatives, se renforcent. Aussi, la part relative des activités des services a basse productivité prend une importance relative plus importante au détriment de celles destinées aux industries de transformation et de services dynamiques, d’où la tendance à la décélération de la productivité du travail à un niveau relativement faible. Cependant cette tendance cache une dispersion relativement forte et plus élevée que par le passé autour de la moyenne. Les grandes entreprises, lorsqu' elles peuvent résister, et parce qu’elles peuvent résister ont des niveaux de productivité et des évolutions beaucoup plus élevées que la moyenne

\section{Graphique: Désindustrialisation précoce en Argentine, Brésil, Chili et Mexique, indice}

100: 1965

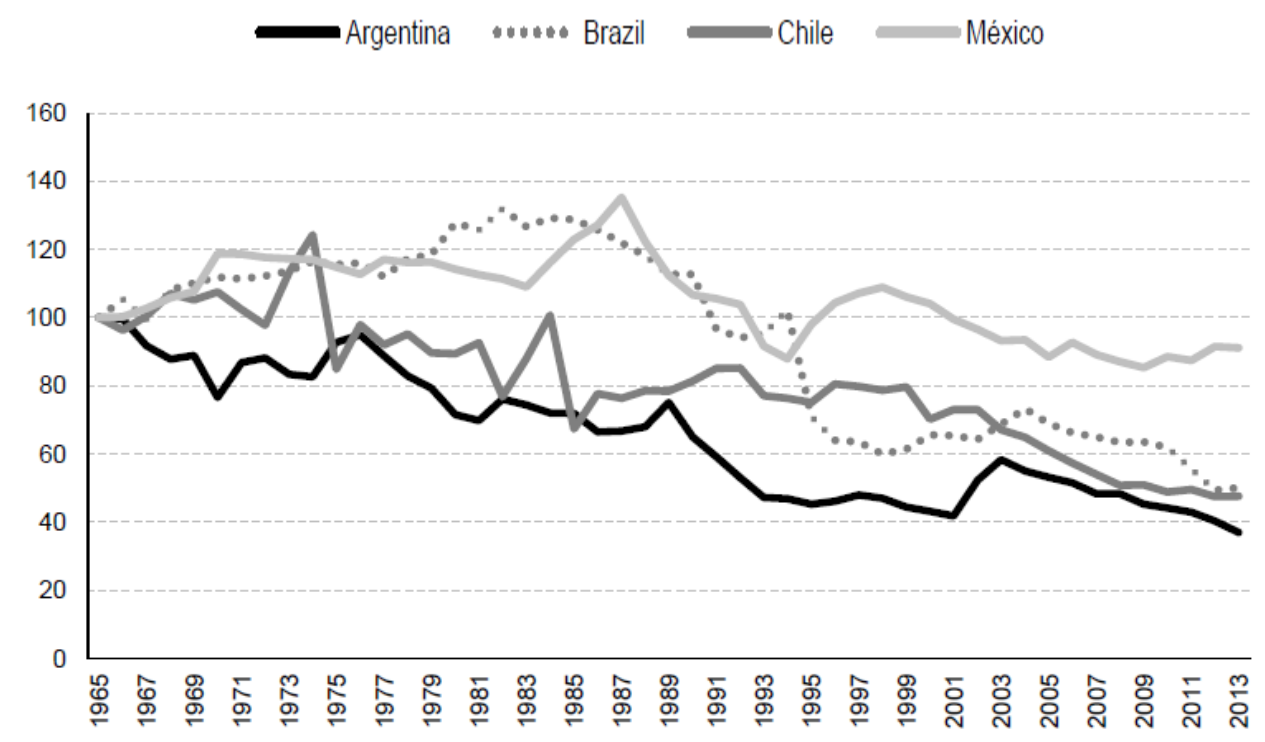

Source: Castillo M. et Martins Neto A. (2016), « Premature desindustrialization in Latin America », Cepal, série Production Development, n²05, 1-23

\footnotetext{
${ }^{14}$ Le poids relatif de l'industrie de transformation régresse au niveau national au profit de ressources naturelles et des services à basse productivité. La rentabilité des capitaux dans les grandes entreprises industrielles baisse et ce dès le début des années 2010 au Brésil, c'est-à-dire avant la baisse du cours des matières premières selon les études du CEMEC (Voir l'ensemble des rapports du CEMEC in http://ibmec.org.br/cemec/notas-cemec/). On observe ce phénomène y compris dans les pays qui ont opté pour l'exportation de produits manufacturés assemblés dans la mesure où leur industrie tournée vers le marché interne devient de moins en moins compétititive.
} 


\section{Graphique : production de l'industrie de transformation au Brésil de janvier 2011 à} janvier 2016

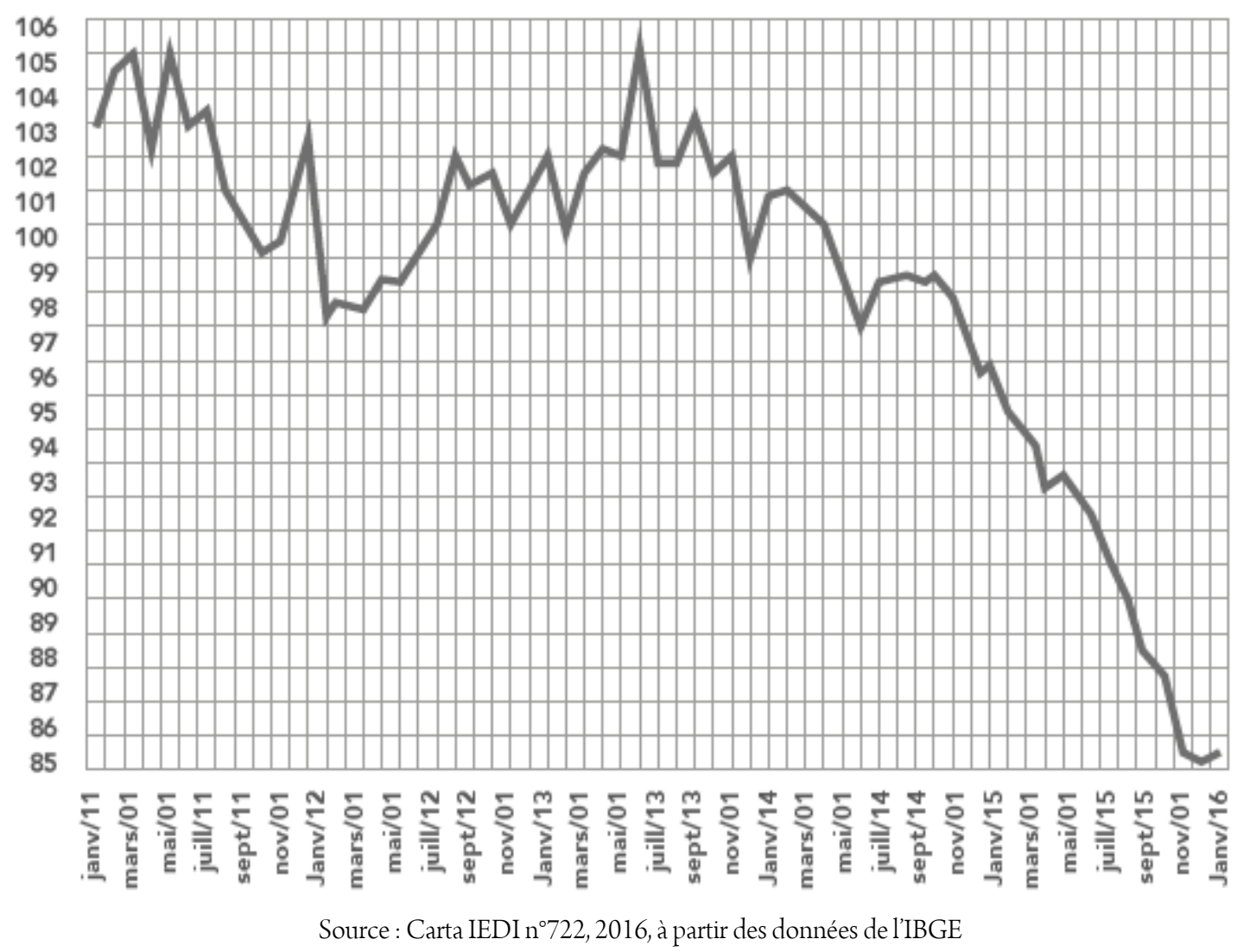

Aujourd'hui, avec le ralentissement économique de la Chine, le cours des matières premières peine a retrouver les cours élevés qu’il avait pu connaitre. La reprimarisation en Amérique latine perd de son dynamisme, sauf au Mexique et en Amérique centrale, où elle est peu présente, ces pays étant peu producteurs de matières premières ${ }^{15}$. L'industrie est affaiblie mais dans quelques pays comme le Brésil, elle n'est pas détruite comme ce fut le cas dans le passé au Venezuela avec le café puis le pétrole. La désindustrialisation qưils connaissent est à la fois précoce et relative ${ }^{16}$. Elle est particulièrement importante, surtout au Brésil, nous venons de le voir. Elle est relative car souvent l'accroissement du déficit en biens de haute et moyenne technologique est davantage le résultat d’une croissance de la demande supérieure à l'offre domestique que d’une régression absolue de celle-ci.

\footnotetext{
${ }^{15}$ Cependant, les remessas (transferts monétaires de leurs travailleurs émigrés aux Etats-Unis) jouent le rôle des matières premières dont ils sont relativement dépourvus. Au Mexique, les remessas se sont élevées à 26 milliards de dollars en 2016 et, ajoutée aux entrés des investissements étrangers directs, ont permis à la fois de «boucler » la balance commerciale de produits industriels légèrement déficitaire et la balance des comptes courants déficitaire, d’augmenter les réserves internationales et, jusqu'à la venue de Trump à la présidence des Etats Unis, elles ont favorisé une appréciation de leur monnaie nationale face au dollar, un peu comme les matières premières au Brésil.

${ }^{16}$ On dit que la désindustrialisation d'un pays émergent est précoce par rapport à la désindustrialisation affectant certains pays avancés lorsque le revenu par tête au début de ce processus correspond à la moitié de celui des pays avancés au moment où débute
} 
Les fortes dépréciations récentes de leur monnaie nationale, les chutes importantes de l'emploi et des salaires dans une moindre mesure, ont montré que les exportations ont pu rebondir après un laps de temps parfois assez long et que leur progression a pu se poursuivre malgré de nouvelles réappréciations. Il reste que cette situation est fragile et ce d'autant plus que les véritables mesures protectionnistes n’ont pas encore été mises en place.

\section{Des possibilités de reprise par le haut ou par le bas?}

L'hypothèse de ce travail est que seule l'industrie, comprise au sens large - incluant donc les services dits dynamiques dont l'essor est consécutifà la révolution informatique - offre des possibilités de sortie par le haut de la crise qui les affecte. Elle seule peut permettre d’assurer une intégration positive dans la division internationale du travail. Mais s'orienter dans cette direction c'est affronter les comportements rentiers, inverser la logique rentière (primarisation et financiarisation) en mettant en œuvre à la fois une réforme fiscale diminuant les inégalités (dynamisant le marché intérieur) et dégageant des ressources pour financer des niches industrielles (politique industrielle non clientéliste), une politique conséquente vis-à-vis de l'éducation et de la recherche qui tout en poursuivant celle qui a été entreprise la développe.

Dans le cadre de cet article, faute de place, nous analyserons surtout le cas emblématique de l'économie brésilienne. Il faut cependant se garder de considérer que ce qui est valable pour le Brésil le serait point par point pour les autres pays. Ils sont plus ou moins différents mais la plupart d'entre eux - à l'exception des pays d'Amérique centrale et du Mexique faute de matières premières abondantes -se sont reprimarisés et tous ont connu une appréciation de leur monnaie face au dollar plus ou moins importante, entrecoupée de fortes dépréciations -dévaluations et une désindustrialisation plus ou moins importante, au Mexique celle-ci étant limitée aux industries tournées vers le marché intérieur.

Comparer les évolutions de la production de l'industrie de transformation selon le degré technologique avec les importations et les exportations est riche d'enseignements. Elle permet d'évaluer avec plus de précision les capacités durables de rebond de l'industrie. Nous verrons que celles-ci existent mais qu'elles sont réduites s'il n'y a pas des modifications substantielles des politiques économiques afin de faire face à la fois à l'héritage (perte de compétitivité et de complexité des exportations) et au protectionnisme qui se développe depuis le début des années 2010, et particulièrement avec le changement de présidence aux Etats-Unis.

leur désindustrialisation. La désindustrialisation concerne non seulement la baisse relative et absolue des emplois industriels, et la destruction d'une partie du tissu industriel, celle produisant notamment les produits de bas de gamme. 
Production et déficit, des données révélatrices

Graphique: Production selon l'intensité technologique, variation annuelle, 2004 - 2017, $1^{\circ}$ trimestre, variations par rapport au trimestre de l'année qui précède, en pourcentage, au Brésil

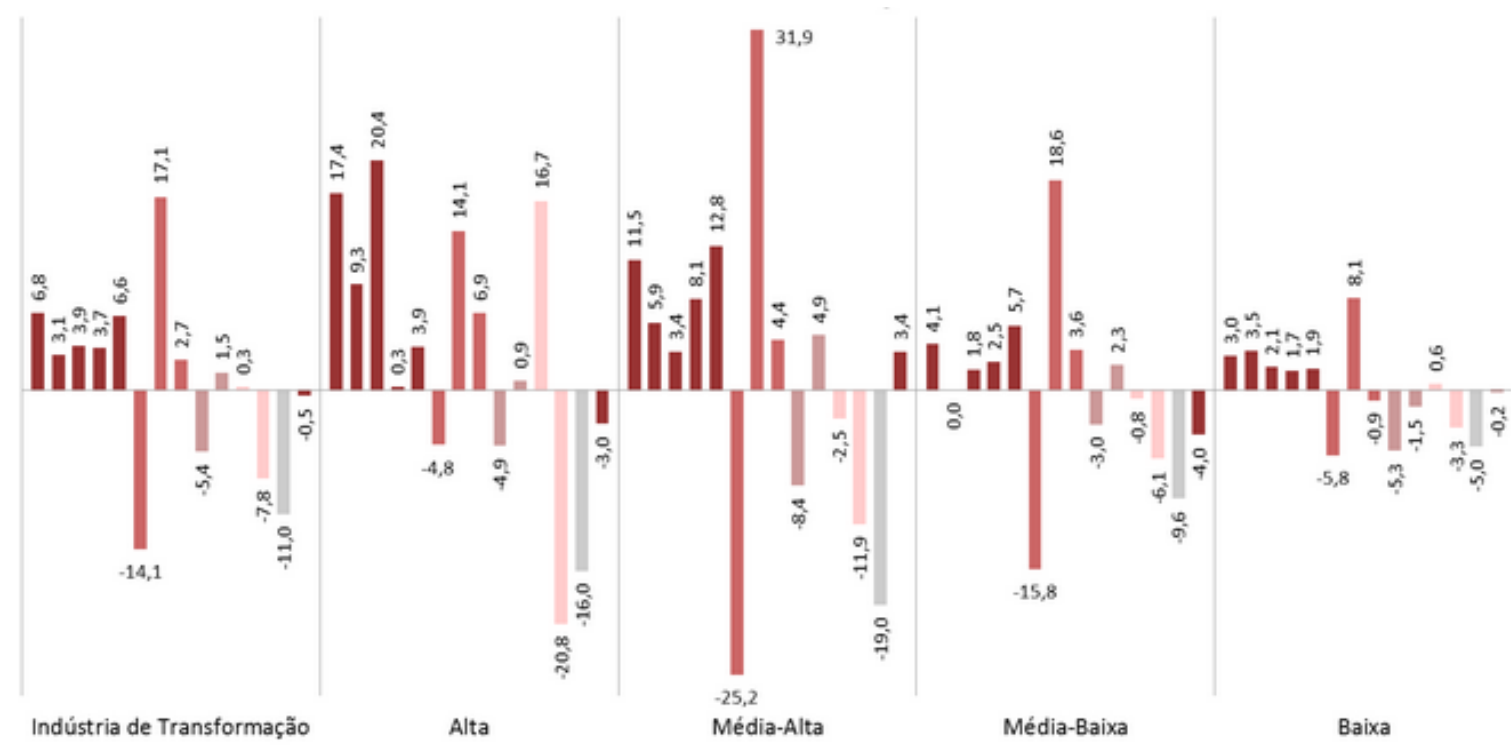

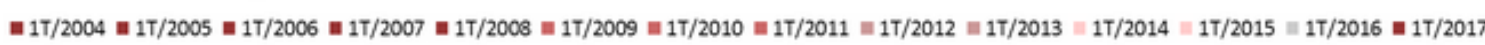

Source: Carta IEDI n788, du 19.05.2017, a partir des données de l’IBGE.

Plus précisément, l'industrie de transformation croit de 2004 à 2008, chute ensuite, se redresse légèrement et s'effondre en 2015 et 2016. Ces mouvements sont amplifiés à la hausse et à la baisse pour les industries de haute technologie (aéronautique et espace, pharmacie, matériel informatique, etc.) et celles de moyenne haute technologie (machines et équipements électriques, véhicules automobiles, produits chimiques sauf pharmacie, machines et équipements mécaniques, etc.). Ce n'est pas le cas pour les autres industries, celles de moyenne-basse (construction, réparation navale, caoutchouc et plastiques, produits métalliques, etc.) et de basse technologie (alimentation, boisson, textile, bois, etc.), cette dernière résistant légèrement mieux à la crise ces dernières années dans la mesure où elle ne s'effondre pas. Elle baisse moins qu'en moyenne l'industrie de transformation. Au total, on observe que les industries de haute technologie et surtout de moyenne -haute technologie connaissent une progression très importante de leur production jusquà la veille de la crise de 2008. Mais cette progression est en deça de celle des importations et donc de la demande domestique. Sur ces produits, la désindustrialisation est donc relative. 
Tableau ${ }^{\circ}$ : Solde de la balance commerciale du Brésil et soldes de la balance commerciale des produits de transformation selon l'intensité technologique, $1^{\circ}$ trimestre 2003 au $1^{\circ}$ trimestre 2017, variation annualisée en millions de dollars FOB.

\begin{tabular}{|c|c|c|c|c|c|c|c|c|c|c|c|c|c|c|c|}
\hline & 2003 & 2004 & 2005 & 2006 & 2007 & 2008 & 2009 & 2010 & 2011 & 2102 & 2013 & 2014 & 2015 & 2016 & 2017 \\
\hline $\begin{array}{l}\text { Solde de } \\
\text { la bc de } \\
\text { l'ind de } \\
\text { trans }\end{array}$ & 2683 & 4086 & 6965 & 6359 & 5495 & -204 & $\begin{array}{c}- \\
2668\end{array}$ & $\begin{array}{c}- \\
7137\end{array}$ & $\begin{array}{c}- \\
10013\end{array}$ & $\begin{array}{c}- \\
13290\end{array}$ & $\begin{array}{c}- \\
16338\end{array}$ & $\begin{array}{c}- \\
18878\end{array}$ & $\begin{array}{c}- \\
14671\end{array}$ & -2006 & -2478 \\
\hline $\begin{array}{l}\text { Autres } \\
\text { (matières } \\
1^{\circ} \text { etc.) }\end{array}$ & 1129 & 2077 & 1482 & 2969 & 3233 & 2961 & 5656 & 8018 & 13155 & 15707 & 11156 & 12739 & 9115 & 10393 & 16896 \\
\hline $\begin{array}{c}\text { Solde de } \\
\text { la bc }\end{array}$ & 3822 & 6162 & 8348 & 9328 & 8728 & 2757 & 2988 & 881 & 3142 & 2418 & -5182 & -6079 & -5557 & 8388 & 14418 \\
\hline $\begin{array}{l}\text { Haute } \\
\text { techo }\end{array}$ & $\begin{array}{c}- \\
1232\end{array}$ & $\begin{array}{c}- \\
1672\end{array}$ & $\begin{array}{c}- \\
1601\end{array}$ & $\begin{array}{c}- \\
2608\end{array}$ & $\begin{array}{c}- \\
3293\end{array}$ & $\begin{array}{c}- \\
4550\end{array}$ & $\begin{array}{c}- \\
3067\end{array}$ & $\begin{array}{c}- \\
6159\end{array}$ & -6804 & -7564 & -7798 & -8336 & -6943 & -4449 & -4764 \\
\hline $\begin{array}{l}\text { Moy- } \\
\text { haute } \\
\text { techno }\end{array}$ & $\begin{array}{c}- \\
1326\end{array}$ & $\begin{array}{c}- \\
1933\end{array}$ & -253 & -86 & $\begin{array}{c}- \\
1365\end{array}$ & $\begin{array}{c}- \\
4843\end{array}$ & $\begin{array}{c}- \\
5754\end{array}$ & $\begin{array}{c}- \\
7491\end{array}$ & $\begin{array}{c}- \\
10991\end{array}$ & $\begin{array}{c}- \\
12079\end{array}$ & $\begin{array}{c}- \\
14736\end{array}$ & $\begin{array}{c}- \\
13863\end{array}$ & $\begin{array}{c}- \\
12306\end{array}$ & -6007 & -6301 \\
\hline $\begin{array}{l}\text { Moy- } \\
\text { basse } \\
\text { techno }\end{array}$ & 1163 & 1522 & 2544 & 2612 & 2515 & 893 & 563 & $\begin{array}{c}- \\
1182\end{array}$ & -710 & -1283 & -2930 & -4224 & -2560 & 271 & -179 \\
\hline $\begin{array}{l}\text { Basse } \\
\text { techno }\end{array}$ & 4082 & 5269 & 6177 & 6436 & 7648 & 8296 & 6400 & 7695 & 8491 & 7637 & 8525 & 7603 & 7139 & 8579 & 8756 \\
\hline
\end{tabular}

Source : élaboration de l'IEDI (Instituto de Estudos para o Desenvolvimento Industrial) à partir de la classification de l'Ocde à partir de la classification de l'OCDE, carta IEDI n 727, avril 2016 et nº 784 du 28 avril 2017

1. La balance commerciale des produits de l'industrie de transformation devient négative en 2008. En 2014 elle atteint son plus grand creux (presque 19 milliards de dollars de déficit). Ce n'est qu’en 2016 - suite à la très forte dépréciation de la monnaie nationale et à l'approfondissement de la crise économique entrainant une réduction des importations et alors même que la monnaie commence à se réapprécierque ce déficit est fortement réduit puisqu’il est divisé par neuf. Ce déficit est largement compensé par la hausse, à la fois, du cours et des volumes échangés des matières premières. Mais cette augmentation considérable ne suffit plus à compenser le déficit fortement croissant de la balance commerciale des produits de l'industrie de transformation dès 2013. A partir de cette date, la balance commerciale globale, d'excédentaire devient déficitaire et ce n'est qu'au premier trimestre 2016 qu’ elle redevient excédentaire.

2. Seule la balance commerciale des produits de basse technologie reste excédentaire tout au long de la période (2003-2017). Le solde de la balance commerciale des produits des haute technologique voit son déficit sextupler de 2003 à 2015 et diminuer fortement ensuite. Le solde la balance des produits de moyenne haute technologie connait un approfondissement considérable de son déficit multiplié par 10 de 2003 à 2015. Ce déficit diminue ensuite de moitié. Enfin le solde de la balance des produits de 
moyenne basse technologie connait un déficit à partir de 2010 mais tend vers l'équilibre à partir de 2016.

\section{$\underline{\text { Un diagnostic relativement pessimiste }}$}

Rester à ce niveau de l'analyse est cependant insuffisant et les conclusions qu'on pourrait en tirer pourraient être trompeuses. De forts déficits peuvent cacher une augmentation de la production, en deça cependant de la demande. Les soldes ne sont que le reflet comptable de l'évolution des importations et des exportations. Ceux sont celles-ci qu’il faut expliquer. Les exportations, classées selon leur niveau technologique, peuvent croitre moins rapidement que les importations, ou bien décroitre. Dans le premier cas, la production locale, bien que croissante, est insuffisante pour des raisons de compétitivité et de rentabilité. Elle n'exporte pas suffisamment et l'excès de demande interne sur la production nette des exportations se reporte sur des importations. Dans le second cas, les entreprises exportent de moins en moins et les importations se substituent en partie à la production locale défaillante. Il convient enfin d’affiner l'analyse au sein des branches: toute production, y compris celle destinée pour partie à l'exportation nécessite des importations et ce d'autant plus qu'avec la l'internationalisation croissante des lignes de production l'éclatement international de la chaine de valeur est plus important qu'hier, même s'il est moins conséquent que dans les pays asiatiques. Aussi, à partir de cet affinement de l'analyse, on peut considérer que le tissu industriel pour chaque branche est plus performant s'il existe la possibilité de monter en gamme et de produire à la fois des produits plus sophistiqués et plus complexes.

A priori le diagnostic qu'on peut faire de l'évolution de l'ensemble du tissu industriel est relativement négatif, mais une analyse plus fine permet de conserver un certain optimisme quant aux capacités de rebond et de réinsertion plus favorable qu'hier dans la division internationale du travail si toutefois certains pré-requis de politique économique sont satisfaits.

Nous avons vu que le solde de la balance commerciale brésilien des produits de l'industrie de transformation devenait négatif dès 2008. Lorsqu’on classe les produits selon leur degré de sophistication technologique, plus le produit est sophistiqué, plus le solde négatif croit. C’est un premier signe à la fois de perte de compétitivité et d'incapacité de produire suffisamment dans les branches de haute technologie, la spécialisation industrielle portant de plus en plus sur des produits de basse technologie. Ceci est confirmé par la perte en avantages comparatifs révélés. Les courbes de l'industrie de transformation déclinent toutes à partir du début des années 2000 en Amérique latine selon le FMI ${ }^{17}$. Telle n'est pas l'évolution que connaissent les économies émergente asiatiques.

\footnotetext{
${ }^{17}$ Xiaodan Ding et Metodij Hadzi-Vaskov (2017) : Composition of Trade in Latin America and the Carabbean, Working paper WP/17/42 IMF
} 
Lorsqu'on décompose la complexité des exportations ${ }^{18}$ en quintiles selon leur complexité croissante et qu’on compare l'évolution de celles-ci, on observe que les économies latino-américaines et les pays asiatiques émergents acquièrent une complexité croissante jusqu’à la fin des années 1990. Ce n’est plus le cas ensuite. Les produits les moins complexes maintiennent relativement leur part en Amérique latine alors qu'ils chutent fortement en Asie émergente. Les quatre autres quintiles, par ordre de complexité croissante, ont une part relative à peu près équivalente au début des années 1960 dans les deux ensembles de pays, soit 80\% et 75\% en 1962. A la fin des années 1990, cette part passe à 60 \% en 1998 puis 52\% en 2012 en Amérique latine alors qu’elle continue à croitre en Asie émergente puisqu’elle se situe à 70\% en 1998 et 80\% en 2012.

\section{Complexité des exportations par quintile Amérique latine et Asie émergentes, 1962-2012}
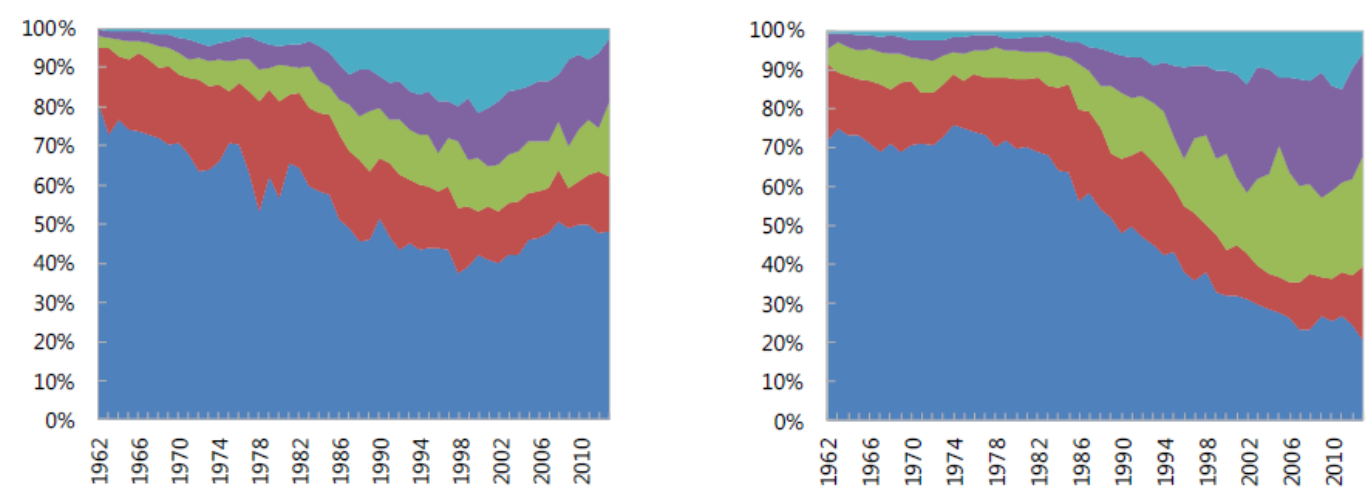

Source : Xiaodan Ding et Metodij Hadzi-Vaskov, p.23

\footnotetext{
${ }^{18}$ Rappelons que la complexité des exportations se mesure en faisant appel à deux propriétés : l'ubiquité et la diversification des exportations. Plus l'effort en recherche développement est important dans un pays, plus sa capacité de produire et d'exporter un ensemble de biens est grande et moins d'autre pays, n'assumant pas le même effort en recherche développement, pourront le produire. La complexité est mesurée par un indicateur qui tient compte et dette diversité en la pondérant par le degré d'ubiquité. Voir infral'encadré.
} 
Parmi les pays appartenant au bloc des BRIC, la complexité décroit fortement à partir de la fin des années 1990 au Brésil et en Russie alors qu'elle s'accroit en Chine et très légèrement en Inde.

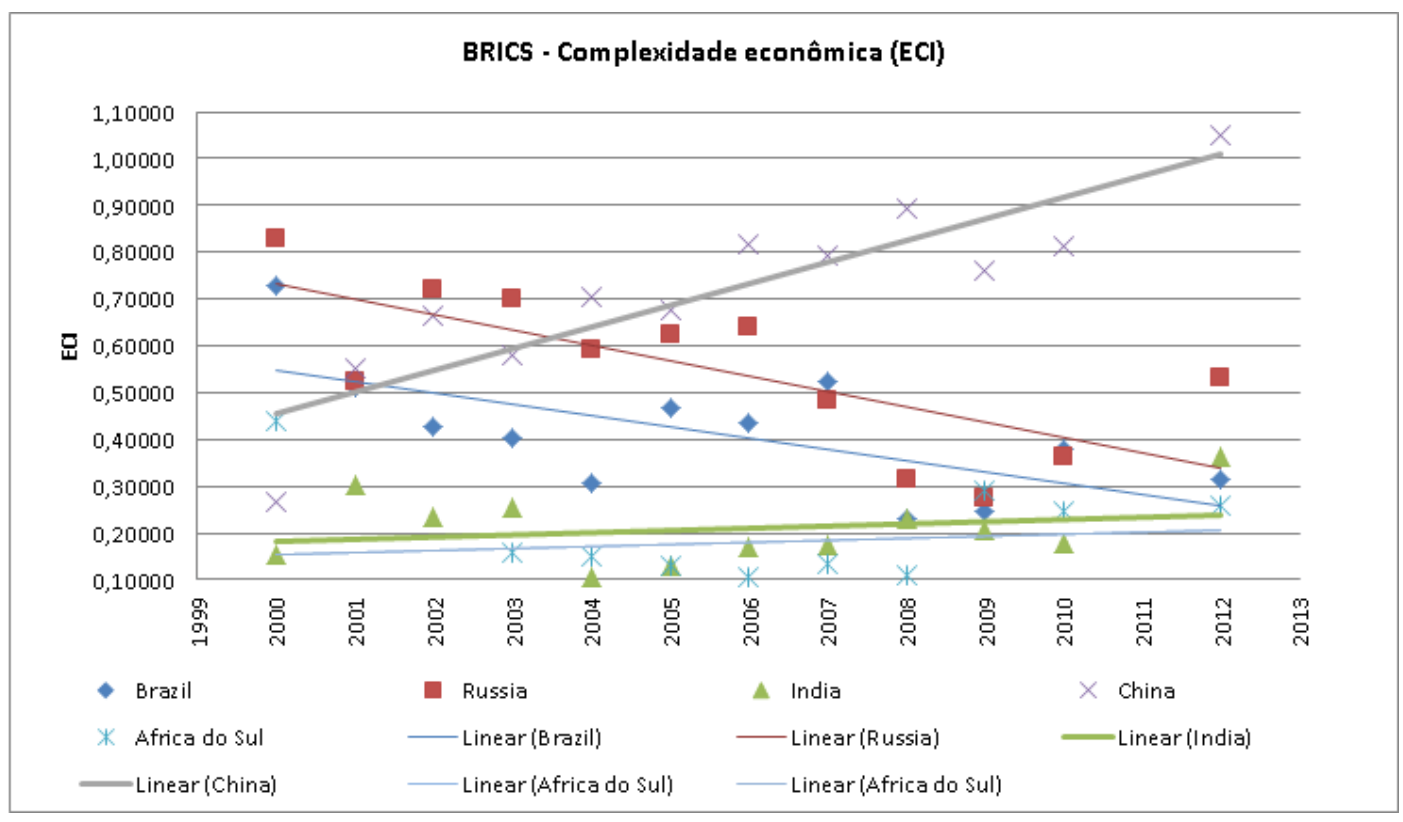

Source: blog Paulo Gala: https://i2.wp.com/www.paulogala.com.br/wp-content/uploads/2015/01/ECI_Brics.png

\section{des raisons d'espérer?}

Désindustrialisation ne signifie pas disparition. A partir de 2015 la plupart des économies latinoaméricaines connaissent une dépréciation de leurs monnaies nationales par rapport au dollar. Au Brésil, cette dépréciation a été très importante. Comme parallèlement, avec la crise, la poussée inflationniste et la montée du chômage, les salaires réels exprimés en monnaie locale ont chuté, le coût unitaire du travail a baissé considérablement malgré une quasi stagnation de la productivité du travail. Au bout de quelques mois, les effets favorables de ce retour vers une certaine compétitivité ont favorisé un essor des exportations au Brésil, malgré la léthargie de la demande mondiale. La part des exportations de l'industrie de transformation dans les exportations totales s'est pour la première fois accrue alors qu'elle avait régressé fortement entre 2005 (53\%) et 2015 (37\%). Le solde de la balance commerciale des produits de l'industrie de transformation est ainsi passé de - 19 milliards de dollars au premier trimestre (annualisé) en 2014 à - 2,5 milliards de dollars au premier trimestre 2017. Entre les mêmes dates, le solde négatif des biens de haute technologie a été divisé par deux, celui des bien de moyennehaute technologie par un peu plus de deux et celui de moyenne basse technologie a quasiment disparu.

Le tissu industriel conserve donc des capacités de réponse à la demande internationale lorsque la compétitivité s'accroit de nouveau. Pour autant cette capacité est fragile car d'une part, elle ne repose pas sur un accroissement de la productivité du travail et d'autre part, elle est le résultat à la fois de la dépréciation d'une 
monnaie sur-appréciée et d’une baisse relative des salaires réels exprimés en monnaie locale. Elle n’est donc pas structurelle, elle est conjoncturelle. Le fait que la monnaie nationale s'apprécie de nouveau depuis fin 2016 et que les salaires réels en monnaie locale ne continuent pas à baisser, voire, commencent à se redresser, constituent un risque majeur que le déficit de la balance commerciale s’accroisse de nouveau.

Ceci dit, si le tissu industriel conserve cette capacité de rebond, c’est parce qu'il n’a pas été complètement détruit: la complexité a diminué, les avantages comparatifs révélés se sont détériorés mais le Brésil conserve encore quelques fleurons. Lorsqu'on met en parallèle les données sur les importations, les exportations de produits industriels selon leur degré technologique et enfin la production domestique de ces mêmes produits, on observe que les industries de haute technologie et surtout de moyenne -haute technologie connaissent une progression très importante de leur production jusqu’à la veille de la crise de 2008. Nous l’avons vu.

Brésil : Importations selon l'intensité technologique, variations annuelles en $\%, 1^{\circ}$ trimestre $2010-1^{\circ}$ trimestre 2017 , en pourcentage, variation annuelles, production (données entre parenthèses)

\begin{tabular}{|c|c|c|c|c|c|c|c|c|}
\hline & 2010 & 2011 & 2012 & 2013 & 2014 & 2015 & 2016 & 2017 \\
\hline Haute & 35.2 & 7.2 & 9.3 & 0.8 & -7.1 & -12.6 & -25.5 & 3.6 \\
technologie & $(14.1)$ & $(6.9)$ & $(-4.9)$ & $(0.9)$ & $(16.7)$ & $(-20.8)$ & $(-16)$ & $(-3)$ \\
\hline Moy HT & 30.9 & 33 & 9.3 & 3.8 & -2.5 & -16.4 & -29.9 & 9 \\
\hline Moy BT & $(31.9)$ & $(4.4)$ & $(-8.4)$ & $(4.9)$ & $(-2.5)$ & $(-11.9)$ & $(-19)$ & $(3.4)$ \\
\hline BT & 62.1 & 21.4 & 18.8 & 5.8 & 2.5 & -16.4 & -41.8 & 29.6 \\
\hline
\end{tabular}

Source: Carta IEDI, n 784, 28/04/2017 et Carta IEDI nº 788 op.cit, voir infra, en grisé, années de crise

A l'inverse, la progression des exportations n'est élevée que sur les produits de moyenne-haute technologie et moyenne-basse technologie, le Brésil étant de moins en moins capable de s'insérer dans la division internationale du travail sur ce secteur. Il reste que dans l'ensemble et par secteurs, il y a à la fois une baisse de la complexité des exportations et des avantages comparatifs en déclin plus ou moins prononcé selon les pays. 


\section{Graphique : Taux de croissance annuel des exportations de l'industrie de transformation}

\section{selon l'intensité technologique}

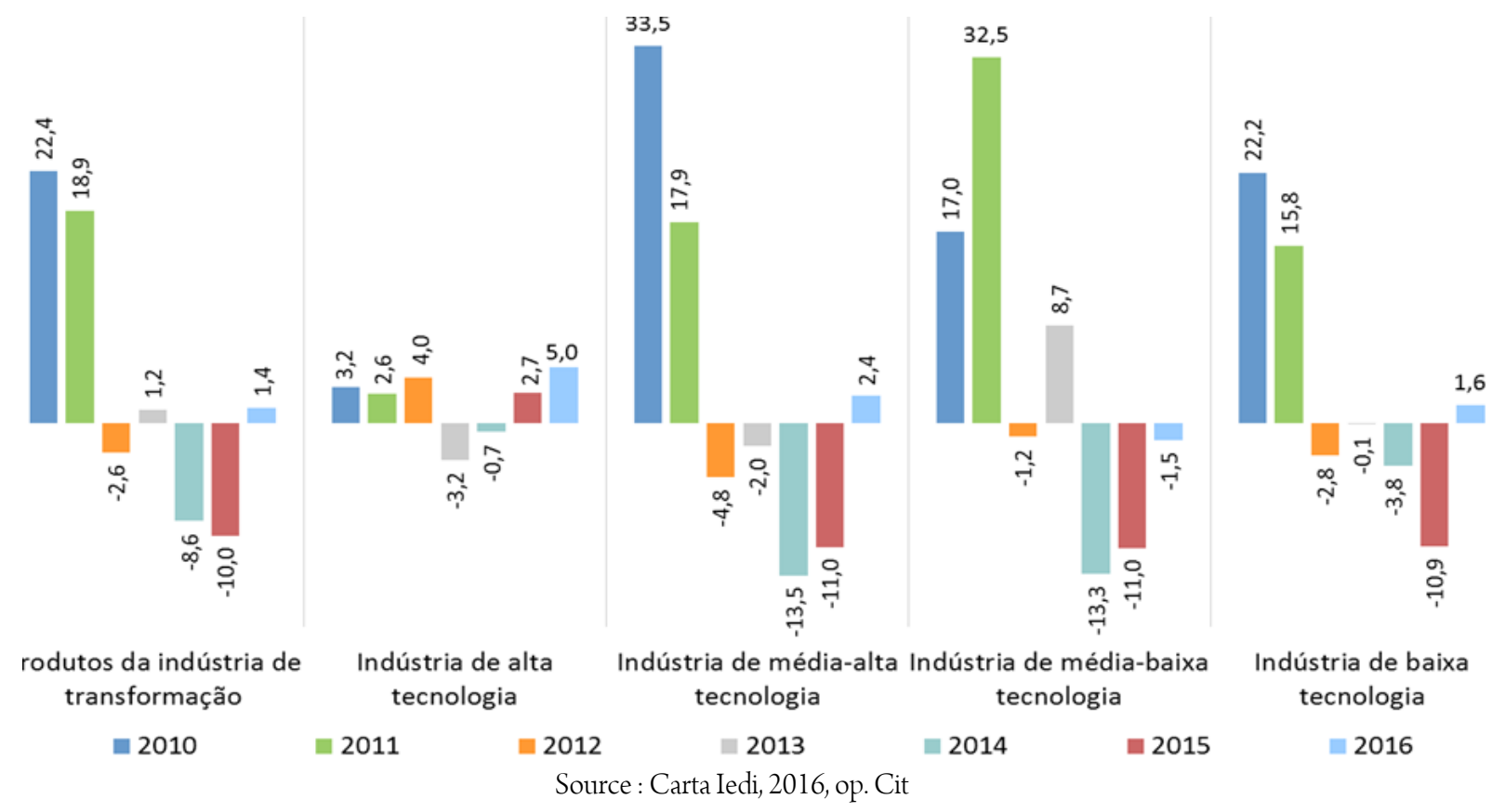

Les taux de croissance des exportations de produits de haute technologie sont relativement faibles mais résistent mieux à la crise que les autres

\section{EN GUISE DE CONCLUSION:}

Les capacités de rebond existent, mais elles sont de plus en plus faibles. Une reprise durable de la croissance est possible. Elle passe par un renforcement des secteurs porteurs d'avenir et non par leur affaiblissement. C'est donc tout le paradigme économique qui a abouti à la fois à la désindustrialisation, à la baisse de la complexité des exportations qu'il faut repenser. Ceci passe par un renforcement de la demande interne et par une compétitivité accrue. Cela semble un oxymore. C’est pourtant la seule voie pour sortir durablement de la crise et ou du ralentissement économique. La réduction des inégalités devrait passer par une réduction des inégalités sociales qui devrait permettre d'impulser la demande. Cette réduction des inégalités pourrait se faire par une réforme fiscale «progressive ». Passer d'un système fiscal régressif à un système fiscal progressif serait une révolution et présuppose que nombre de conflits soient résolus. C'est dire la difficulté. Pour éviter que l'accroissement de la demande conduise à une augmentation des importations, il faudrait à la fois dévaluer la monnaie, empêcher qu’elle se réapprécie par des mécanismes de stérilisation, et définir une politique industrielle qui permette d'accroitre la productivité du travail. Le chemin est escarpé, c’est le seul possible.

De ce point de vue, le ralentissement des échanges internationaux, la montée des protectionnismes, peuvent être une opportunité pour opter pour un autre modèle de développement et connaitre une croissance 
durable qui respecte davantage les citoyens et l'environnement. L'Histoire enseigne qu'elle avance par rupture. Les difficultés que connaissent la plupart des pays latino-américains n’ont pas de solutions qu'économiques. De nouvelles alliances de classes peuvent apparaitre, comme dans les années trente et permettre un rebond économique de ces pays. Mais ceci est une toute autre histoire...

\title{
CAN A CHANGE OF PACE OF GLOBALIZATION BE AN OPPORTUNITY FOR EMERGIN LATIN AMERICANS?
}

\begin{abstract}
The article analyzes the recent growth of international trade in goods and services and the greater openness of countries to international trade and the end of globalization since 2008, with the resumption of protectionist policies in several countries, especially the United States. In this context, it warns that the slowdown in international trade and the rise of protectionism may be an opportunity for Latin American emerging countries to opt for a different model of development and more sustainable growth that respects citizens and the environment. He says that in this context, new class alliances can occur, as in the 1930s, and allow economic recovery in these countries, in the ebb of globalization.
\end{abstract}

Keywords: Reflux of globalization; Protectionist policies; Another model of sustainable development; New class alliances; Economic recovery.

\section{RÉFÉRENCES}

BALDWIN, R. (2016), The Great convergence, Information, Technology end the New globalization, Havard: Harvard University press, 2016.

DING, Xiaodan et HADZI-VASKOV, Metodij. (Composition of Trade in Latin America and the Carlbbean. Working paperWP/17/42 IMF, 2017.

FAJNZYLBER, F. Industrializacion e internationalizacion de la America Latina. México: Fundo de Cultura economica, 1980.

HIRATUKA et SARTI. Relações econômicas entre Brasil e China: análise dos fluxos de comércio e investimento direto estrangeiro. Revista Tempo do Mundo, Brasil, IPEA, vol 2 n 1, pp.83-98, 2016.

OCDE (Organisation de coopération et de développement économiques), CAF (Corporacion Andina de Fomento) et CEPAL. Towards a new partnership with China. Latin American Economic Outlook, 2016.

OCDE (Organisation de coopération et de développement économiques et CEPAL. Perspectives économiques de l'Amérique latine, transformation de l'Etat et développement, 2012. 
RIFKIN, J. La nouvelle société du coût marginal zéro, L'internet des objets, l'émergence des communaux collaboratifs et l'éclipse du capitalisme, Paris: Édition Babel, 2016.

ROMERO TELLAECHE, J. A. Los limites al crescimiento economico de Mexico, México: El Colegio de Mexico/Universidad Nacional Autónoma de Mexico, 2014.

SALAMA, Pierre. Amérique Latine, des années 1950 à aujourd'hui, mutations, essor et nouvelles dépendances. Paris, Revue d'économie financière, $n^{\circ} 124,23-44,2012$.

Les économies émergentes latino-américaines, entre cigales et fourmis. Paris: Armand Colin, 2012.

SASSEN, S. The Global City: New York, London, Tokyo. Princeton: Priceton University Press, 1991.

VELTZ, P. La société hyper-industrielle, le nouveau capitalisme productif. Paris: Édition du Seuil, 2017.

Trabalho enviado em 09 de julho de 2017.

Aceito em 17 de julho de 2017. 